\title{
Topic 11: Distributed and High-Performance Multimedia
}

\author{
Frank Seinstra ${ }^{\star}$, Nicolás Guil ${ }^{\star}$, Zoltan Juhasz ${ }^{\star}$, and Simon Wilson
}

In recent years, the world has seen a tremendous increase in the capability to create, share and store multimedia items, i.e. a combination of pictorial, textual, and auditory data. Moreover, in emerging multimedia applications, generation, processing, storage, indexing, querying, retrieval, delivery, shielding, and visualization of multimedia content are integrated issues, all taking place at the same time and - potentially - at different administrative domains.

As a result of these trends, a number of novel and hard research questions arise, which can be answered only by applying techniques of parallel, distributed, and Grid computing. The scope of this topic embraces issues from highperformance processing, coding, indexing, and retrieval of multimedia data over parallel architectures for multimedia servers, databases and information systems, up to highly distributed architectures in heterogeneous, wired and wireless networks.

This year 3 papers were submitted to this topic area. All the papers were reviewed by 4 referees, and 2 papers were ultimately selected. A third paper, which was initially submitted to Topic 5 (on Parallel and Distributed Databases), has been included as well, as its contents perfectly fit into the current topic.

The first paper, by S. Khanfir and M. Jemni, presents two new parallel FPGAbased implementation schemes for the lifting-based Discrete Wavelet Transform. The first algorithm uses pipelining, parallel processing, and data reuse for increased execution speed. The second algorithm uses a dynamically reconfigurable controller that determines the optimal number of clones of the first algorithm to be deployed for fastest execution. The authors show the two approaches to allow for real-time processing of large and multi-framed images.

The second paper, by R. Godoi, X. Yang, and P. Hernández, gives an extensive analysis of the requirements of the control subsystem of a peer-to-peer (P2P) multicast architecture for Large Video on Demand (LVoD) service provisioning. The analysis, performed by means of a set of analytical models, considers many different control system design schemes. The results indicate that the control modules in LVoD infrastructures are becoming increasingly important for reasons of scalability, load balance, and server resource utilization.

The third paper, by M. Marin, V. Gil-Costa, and C. Bonacic, presents a novel distributed index data structure, as well as a set of related algorithms, devised to support parallel query processing of multimedia content in high-performance search engines. The presented methodology offers high locality in terms of data accesses, thus allowing for a friendly use of secondary memory, and a high

\footnotetext{
^ Topic Chairs.
} 
potential for exploiting multithreading. Results obtained on a 32-node compute cluster show superior results in comparison with existing approaches.

To conclude, we would like to express our gratitude to the people whose contributions made the Distributed and High-Performance Multimedia track possible. Above all, we thank the authors who submitted a paper, the Euro-Par Organization Committee, and the numerous referees, whose excellent work was an invaluable help for the topic committee. 